\title{
Pilot Attempt to Benchmark Spatial Resolution of an Electronic Imaging System Prototype for In-Process Electron Beam Melting Monitoring
}

\author{
Authors: Hay Wong ${ }^{a}$, Derek Neary ${ }^{\mathrm{a}}$, Eric Jones, Peter Fox ${ }^{\mathrm{a}}$, Chris Sutcliffe ${ }^{\mathrm{a}}$

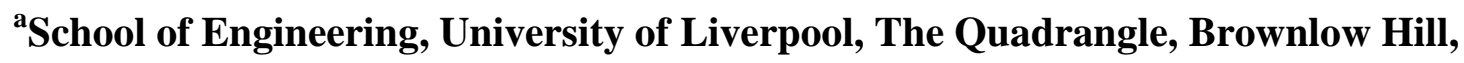 \\ United Kingdom L69 3GH
}

Corresponding author's email: Hay Wong - hay.wong@liv.ac.uk

Keywords: Electron Beam Melting; In-Process Monitoring; Quality Control; Electronic Imaging; Spatial Resolution

\begin{abstract}
Electron Beam Melting (EBM) is an increasingly used Additive Manufacturing (AM) technique employed by many industrial sectors, including the medical device and aerospace industries. In-process EBM monitoring for quality assurance purposes has been a popular research area. Electronic imaging has recently been investigated as one of the in-process EBM data collection methods, alongside thermal/ optical imaging techniques. Despite certain capabilities of an electronic imaging system have been investigated, experiments are yet to be carried out to benchmark one of the most important features of any imaging systems - spatial resolution. This article addresses this knowledge gap by: (1) proposing an indicator for the estimation of spatial resolution which includes the Backscattered Electrons (BSE) information depth, (2) estimating the achievable spatial resolution when electronic imaging is carried out inside an Arcam A1 EBM machine, and (3) presenting an experimental method to conduct edge resolution evaluation with the EBM machine. Analyses of experimental results indicated that the spatial resolution was of the order of $0.3 \mathrm{~mm}-0.4 \mathrm{~mm}$ when electronic imaging was carried out at room temperature. It is believed that by disseminating an analysis and experimental method to estimate and quantify spatial resolution, this study has contributed to the on-going quality assessment research in the field of in-process monitoring of the EBM process.
\end{abstract}




\section{Introduction}

\subsection{Introduction to Electron Beam Melting (EBM)}

EBM is an Additive Manufacturing (AM) technique that makes use of an accelerated electron beam to melt metallic powder on a layer-by-layer basis, forming components based on the geometries of the imported three dimensional (3D) Computer Aided Design (CAD) models [1]. When the electron beam raster-scans across a preheated powder bed in a predefined pattern, melt tracks are solidified and fully-dense cross-sections of the desired component are formed. This process is repeated with the additional requirement that the underlying solid is also partially re-melted to ensure adequate bonding between the underlying and newly formed layers ensuring that full density is achieved in components. The EBM process offers reduced thermal residual stress in components [2] and high level of design freedom [3]. It is thought that the technique shows great promise in the manufacture of orthopaedic implants and aerospace components [4]. However, due to process issues including non-uniform powder layer deposition [5], peeled-off metallisation on the processing area [6], and component defects including, porosity within melted area [5], balling along melt tracks [7] and delamination of processed layer [8], the widespread adoption of EBM in industry is hindered unless a rigorous EBM process monitoring and validation system is available for real-time control [9].

\subsection{Technology Gaps in Electronic Imaging Spatial Resolution Analysis in EBM Monitoring}

Two academic research groups have attempted to employ feedback electronic imaging as the data collection method for monitoring the EBM process. At the Friedrich-AlexanderUniversity of Erlangen-Nürnberg (Germany), Arnold et al [10] introduced a Backscattered Electrons (BSE) imaging system. A BSE detector was mounted inside the electron beam column of a modified Arcam S12 EBM machine (Arcam AB / GE Additive, USA), above the EBM processing chamber. Electronic images were generated from the BSE signals originated from elastic collision between the incident machine electron beam and the processing area. Detection of in-layer porosity within a set of EBM manufactured components were carried out via electronic image analysis. Moreover, results were compared with optical microscopy and X-ray Computed Tomography (CT) images generated post-process, in order to validate the observations from the in-process electronic images. The second group working in the same field is from the University of Liverpool (UK). Wong et al [11] developed a custom 
electronic imaging system prototype and interfaced it with an Arcam A1 EBM machine (Arcam AB / GE Additive, USA). The prototype consisted of a SE/ BSE sensor, signal amplifier, data-logger, and a piece of image generation software [11]. The group investigated the prototype capabilities, and presented findings including, magnification range, the selection of image Field-of-View (FOV) [12], and the influence of temperature on the quality of electronic images [13]. In spite of the research efforts made by these two groups, no information is available regarding (1) the achievable spatial resolution, and (2) how to quantify and benchmark this parameter for an electronic imaging system operating inside an EBM machine. This study aims to address these knowledge gaps by estimating and evaluating experimentally the achievable spatial resolution of an electronic imaging system prototype when interfaced with an EBM machine.

\subsection{Spatial Resolution Evaluation for High Energy Beams}

Spatial resolution is an important feature of an imaging system as it dictates how capable a system can reveal topographical details of an imaging target [14]. Reimer [15] stated that electron beam size and resolution are inter-related in electron microscopy, and spatial resolution can only be worse but not better than the machine electron beam size. Thus, this study postulates that the beam size can serve as an indicator for the estimation of the achievable spatial resolution. There are various ways to define the size of a high energy beam, i.e. laser, electron beam, which includes but not limited to, Full Width Half Maximum

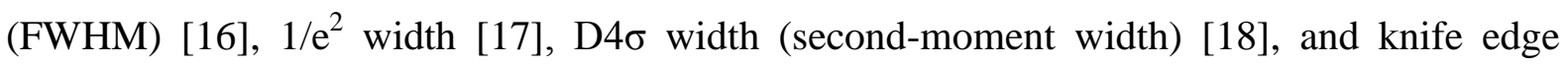
width $[19,20]$. In this pilot study, owing to the simplicity of the experimental setup, the knife edge resolution has been selected as the definition of the spatial resolution for an electronic imaging system interfaced with an EBM machine. In a knife edge test, when a high energy beam moves perpendicularly to and across a sharp edge, for example as shown in Fig. 1(a), indicated by the red dotted lines, the beam intensity spatial distribution profile, i.e. the Edge Spread Function (ESF), illustrated in Fig. 1(b) is observed [15]. The knife edge spatial resolution of the beam can be defined as the spatial width measured between two beam intensity values of $25 \%$ and $75 \%$ of the maximum intensity value measured [15]. 


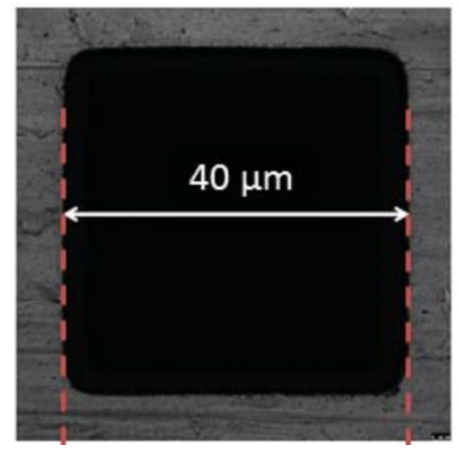

(a) TEM grid with $40 \mu \mathrm{m}$ hole [21]

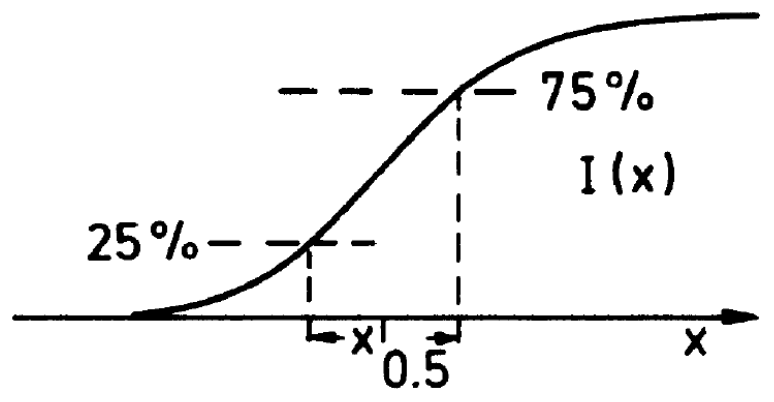

(b) Resolution is the spatial width of values $25 \%$ to $75 \%$ of maximum value [15]

Fig. 1 Sharp edge and the definition of spatial resolution from an ESF

\section{Materials and Methods}

This section first presents the estimation on spatial resolution, and then disseminates the experimental setup for the investigation of spatial resolution in details.

\subsection{Theoretical Analysis of Spatial Resolution}

This article postulates that for the evaluation of spatial resolution in electronic imaging, in addition to the electron beam diameter, the extra width incurred from the feedback electron information depth should also be taken into account. In electronic imaging, the feedback electron signal consists of Secondary Electrons (SE) and Backscattered Electrons (BSE), as shown in Fig. 2(a) [15]. These electrons are generated from the imaging target at different information depths, with BSE originating from deeper inside the imaging target, as indicated by the electron-specimen interaction volume in Fig. 2(b).

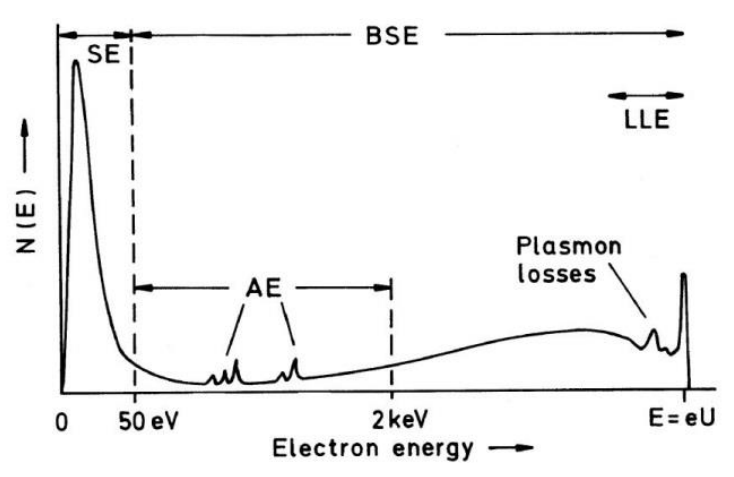

(a) Energy spectrum of feedback electrons

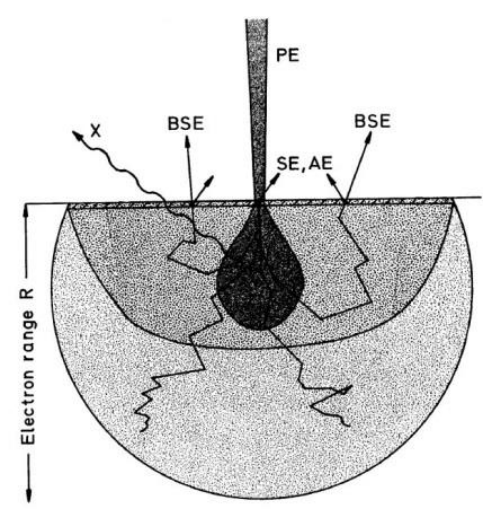

(b) Interaction volume, information depth

Fig. 2 Electron-material interaction and products [15] 
In this study, the spatial resolution of an electronic imaging system is defined as the diameter of the Arcam A1 EBM machine electron beam with the maximum additional width incurred from the information depth, i.e. the BSE information depth. Fig. 3 illustrates this concept and the definitions of beam size and spatial resolution.

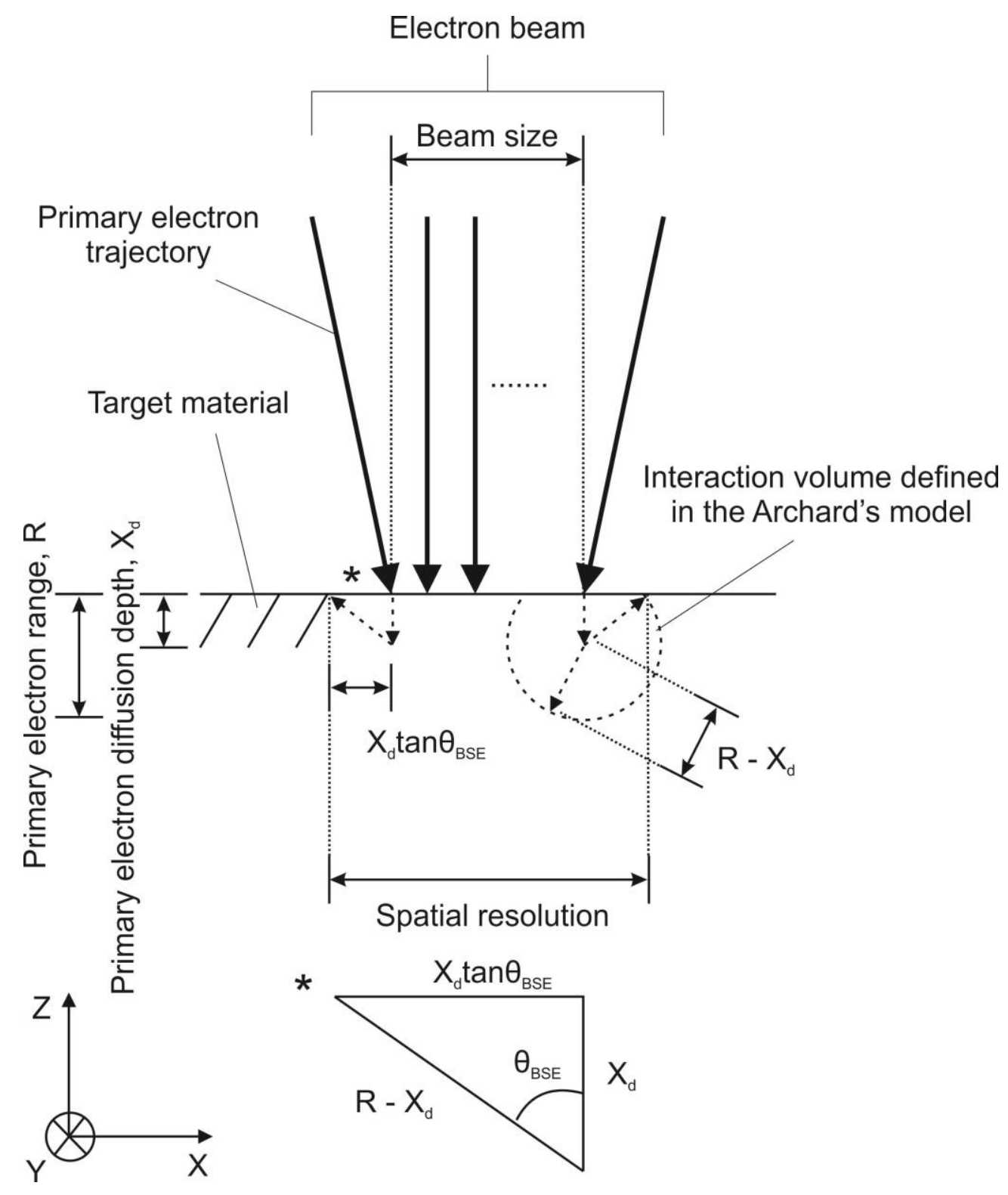

Fig. 3 Beam size and spatial resolution (without gas)

The definition of spatial resolution depicted in Fig. 3 makes use of the concept of BSE generation from Archard's model [22]. This model estimates that, the widest possible angle between the trajectory of a returning BSE and the normal of the target material's surface, is limited by the returning path length, $R-X_{d}$. This is the longest possible path taken by any BSE if it is to have sufficient energy to re-emerge from the target material (BSE energy is 
consumed by inelastic collisions within the material). Table 1 summarises the estimation on spatial resolution using Eq. 1 [22] with titanium being the imaging target material.

$$
\frac{X_{d}}{R}=\frac{40}{7 Z}
$$

Where

$\mathrm{X}_{\mathrm{d}}(\mu \mathrm{m})$ is the electron diffusion depth (complete electron diffusion occurs beyond this point), and $\mathrm{R}(\mu \mathrm{m})$ is the electron penetration range, and $\mathrm{Z}$ is the atomic number of the imaging target

Table 1 Spatial resolution estimation (without gas). Data rouned to 3 s.f.

\begin{tabular}{cc}
\hline Parameter & Value \\
\hline $\mathrm{Z}$ (titanium) $(\mu \mathrm{m})$ & 18.0 \\
$\mathrm{X}_{\mathrm{d}}(\mu \mathrm{m})$ & 4.70 \\
$\mathrm{X}_{\mathrm{d}}$ tanӨBSE $(\mu \mathrm{m})$ & 12.0 \\
Width of BSE region affecting resolution, & \\
Minimum electron beam size (mm) & $0.200[23]$ \\
Spatial resolution without gas, $\mathrm{R}_{\mathrm{sp}(\mathrm{mogs})}(\mathrm{mm})$ & 0.224 \\
\hline
\end{tabular}

The spatial resolution analysis described above was estimated for situations with the absence of gas. However, the Arcam A1 EBM machine operates under helium gas at $2 \times 10^{-3} \mathrm{mbar}$ [24]. The influence of gas on spatial resolution is thought to be the broadening of the primary machine electron beam. This phenomenon is also known as "beam skirting" [25]. It is due to scattering of the electron beam by the gas molecules. The extent of skirting depends on parameters including, primary electron energy, type of gas, gas pressure, beam working distance, and gas temperature. Fig. 4 gives the definition of beam skirt radius [26] whilst Eq. 2 predicts the skirt radius [25]. Table 2 summarises the estimation on skirt radius when electronic imaging is conducted inside the A1 EBM machine at room temperature. 


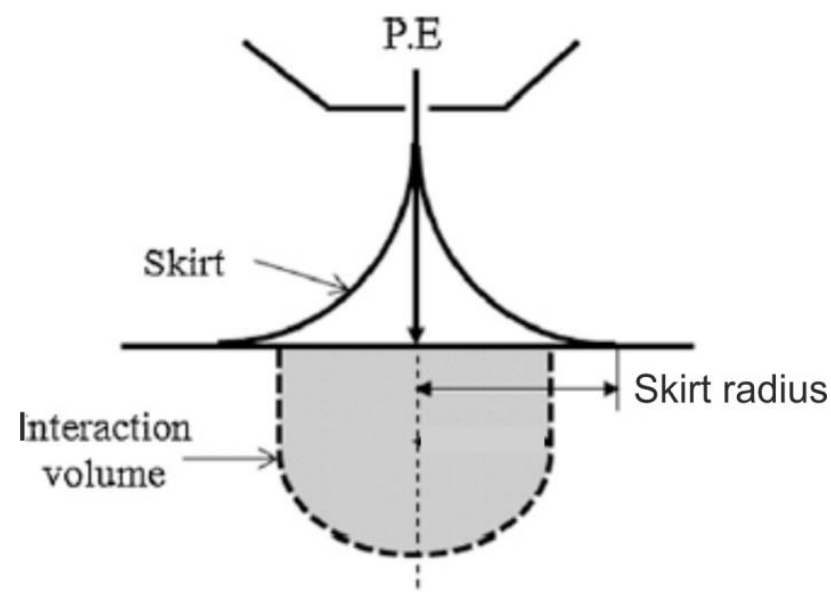

Fig. 4 Definition of electron beam skirt radius, adapted from [26]

$$
r_{\text {skirt }}=\frac{364.19 Z}{E}\left(\frac{P}{T}\right)^{\frac{1}{2}} L_{W . D^{\frac{3}{2}}}
$$

Where

$\mathrm{r}_{\text {skirt }}(\mathrm{m})$ is the beam skirt radius, $\mathrm{E}(\mathrm{eV})$ is the primary electron beam energy, $\mathrm{Z}$ is the atomic number of the gas present, $\mathrm{P}(\mathrm{Pa})$ is the pressure of the gas, $\mathrm{T}(\mathrm{K})$ is the gas temperature, and $\mathrm{L}_{\mathrm{W} . \mathrm{D}}(\mathrm{m})$ is the working distance

Table 2 A1 EBM machine electron beam skirt radius estimation. Data rounded to 3 s.f

\begin{tabular}{cc}
\hline Parameter & Value \\
\hline $\mathrm{Z}$ (helium) $(\mathrm{keV})$ & 60.0 \\
$\mathrm{P}(\mathrm{mbar})$ & $2.00 \times 10^{-3}$ \\
$\mathrm{~T}\left({ }^{\circ} \mathrm{C}\right)$ & 30.0 \\
$\mathrm{~L}_{\mathrm{W} . \mathrm{D}}(\mathrm{mm})$ & 400 \\
$\mathrm{r}_{\text {skirt }}(\mathrm{mm})$ & 0.0789
\end{tabular}

Table 1 gives an estimated spatial resolution of $0.224 \mathrm{~mm}$, when electronic imaging is conducted on a titanium target with the absence of gas. The final spatial resolution with the influence of gas taken into account is calculated by Eq. 3. Table 3 summarises the estimation 
of the achievable spatial resolution of electronic imaging when conducted inside the A1 EBM machine at room temperature.

$$
R_{s p}=R_{s p(\text { no gas })}+2 r_{\text {skirt }}
$$

Where

$R_{s p}(\mathrm{~mm})$ is the final spatial resolution, $R_{s p \text { (no gas) }}(\mathrm{mm})$ is the spatial resolution without gas, and $r_{\text {skirt }}(\mathrm{mm})$ is the skirt radius due to the presence of helium gas at $2 \times 10^{-3}$ mbar.

Table 3 Electron beam spatial resolution estimation (with gas). Data rounded to 3 s.f

\begin{tabular}{cc}
\hline Parameter & Value \\
\hline $\mathrm{R}_{\text {sp(no gas })}(\mathrm{mm})$ & 0.224 \\
$\mathrm{r}_{\text {skirt }}(\mathrm{mm})$ & 0.0789 \\
$\mathrm{R}_{\mathrm{sp}}(\mathrm{mm})$ & 0.382 \\
\hline
\end{tabular}

\subsection{Spatial Resolution Experimental Setup}

Spatial resolution was investigated by conducting edge resolution tests [15] with the use of electronic images generated by a custom digital electronic imaging system prototype developed for an Arcam A1 EBM machine [11]. Fig. 5 is the schematic of the prototype. The prototype consisted of a feedback electron sensor (modified Arcam heat-shield frame and plates), a data logger (Arduino DUE microcontroller break-out board), a signal amplifier and electronic image generation software. 


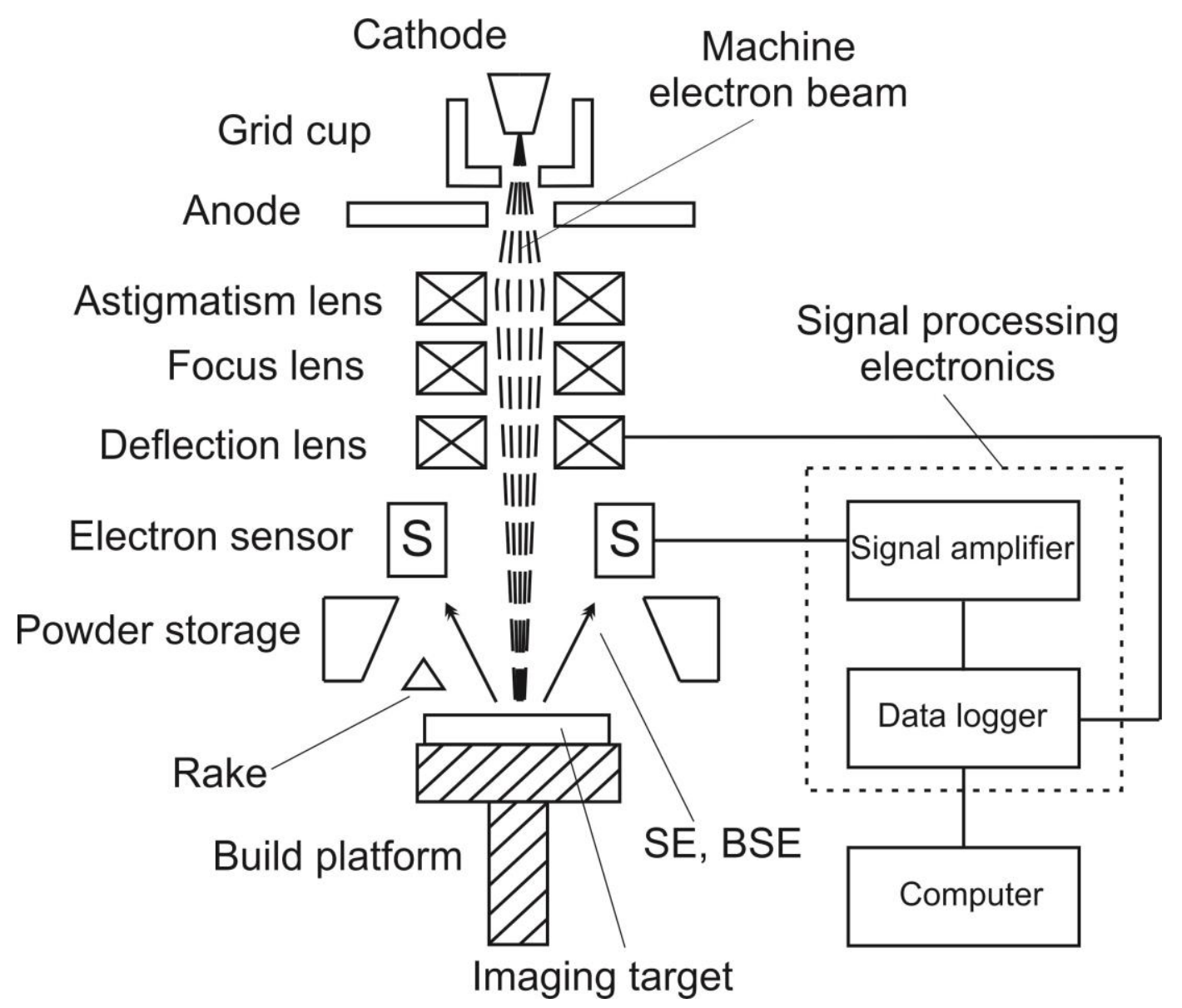

Fig. 5 Electronic imaging system prototype schematic [11]

In the experiments, the electron beam size was evaluated and used as an indicator for spatial resolution. A Grade 316 stainless steel plate (Merseyside Metal, UK) with 3M 1436 aluminium foil tape (3M, USA) applied, as shown in Fig. 6, was used as an imaging target in the experiment. The aluminium tape was used firstly, to create material contrast from the stainless steel plate, due to the sensitivity of feedback BSE signal yield on the atomic number of materials [27], and secondly, the edge of the tape was used to evaluate the electron beam size when the beam scans in a direction perpendicular to the tape edge. The custom electronic imaging system prototype was interfaced with the Arcam A1 EBM machine. Table 4 and 5 summarise the electronic image settings and the configurations of both the EBM machine and the prototype. 
FOVs: $180 \mathrm{~mm} \times 180 \mathrm{~mm} \& 60 \mathrm{~mm} \times 60 \mathrm{~mm}$

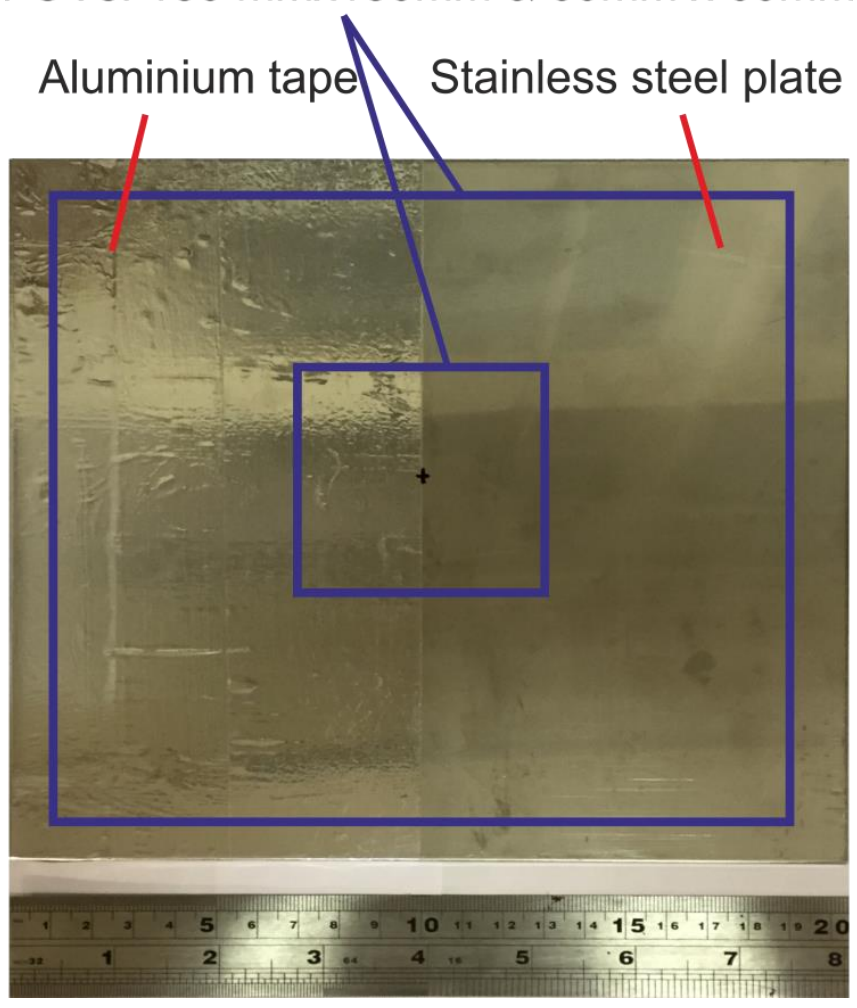

Fig. 6 Imaging target of the spatial resolution experiment

Table 4 Experiment image settings

\begin{tabular}{cccc}
\hline Image Size (pixel) & $\begin{array}{c}\text { Number of Image to } \\
\text { Generate }\end{array}$ & FOV $\left(\mathrm{mm}^{2}\right)$ & $\begin{array}{c}\text { Theoretical pixel } \\
\text { Resolution (mm/pixel) }\end{array}$ \\
\hline $1800 \times 1800$ & 5 & $180 \times 180$ & 0.1 \\
$1800 \times 1800$ & 5 & $60 \times 60$ & 0.033 \\
\hline
\end{tabular}


Table 5 Arcam A1 EBM machine and imaging prototype configurations

\begin{tabular}{|c|c|}
\hline Parameter & Value \\
\hline \multirow{2}{*}{ Beam current (mA) } & 1 (FOV:180x180) \\
\hline & 0.5 (FOV:60x60) \\
\hline Beam scan speed $\left(\mathrm{mms}^{-1}\right)$ & 11880 \\
\hline Chamber pressure (mbar) & $2 \times 10^{-3}$ \\
\hline Signal amplifier gain & 10 \\
\hline Data logger input / output range (V) & 0 to +3.3 \\
\hline Data logger sampling frequency $(\mathrm{Hz})$ & $118.8 \mathrm{k}$ \\
\hline Data logger sampling bit rate (bps) & $950.4 \mathrm{k}$ \\
\hline Imaging area $\left(\mathrm{mm}^{2}\right)$ & $180 \times 180 / 60 \times 60$ \\
\hline \multirow{2}{*}{ Raster-scan spacing (mm) } & 0.1 (FOV:180x180) \\
\hline & 0.033 (FOV:60x60) \\
\hline Image bit depth & 256 \\
\hline Image frame time (s) & 27.3 \\
\hline
\end{tabular}

Once the raw electronic images were generated, pixel value profiles of four scan lines were extracted from each image for spatial resolution analysis, as detailed in Table. 6. The central row (pixel row index: 900) of each image was deliberately avoided due to the damage on the aluminium tape incurred during one of the EBM machine setup procedures, i.e. beam centring. The spatial resolution of the electronic imaging system prototype was determined from the pixel value profile, based on the definition given in Fig. 1(b). 
Table 6 Spatial resolution analysis scan line location

\begin{tabular}{cc}
\hline Scan Line & Pixel Row Index \\
\hline 1 & 300 \\
2 & 600 \\
3 & 1200 \\
4 & 1500 \\
\hline
\end{tabular}

\section{Results}

A total of ten raw electronic images were generated with FOV of $180 \mathrm{~mm} \times 180 \mathrm{~mm}$ and $60 \mathrm{~mm} \times 60 \mathrm{~mm}$. Fig. 7(a) shows the typical raw image with the four scan lines to be extracted for edge resolution analysis. Fig. 7(b) shows the typical pixel value ESF of an extracted scan line from the raw image, and the definition of spatial resolution, based on the definition given in Fig. 1(b). The pixel value data from the raw electronic images was best-fitted from linear regression [28] and the use of a modified standard hyperbolic tangent function, i.e. $\tanh (\mathrm{x})$, as detailed in Eq. 4. Image spatial resolution was then calculated based on Eq.5. 


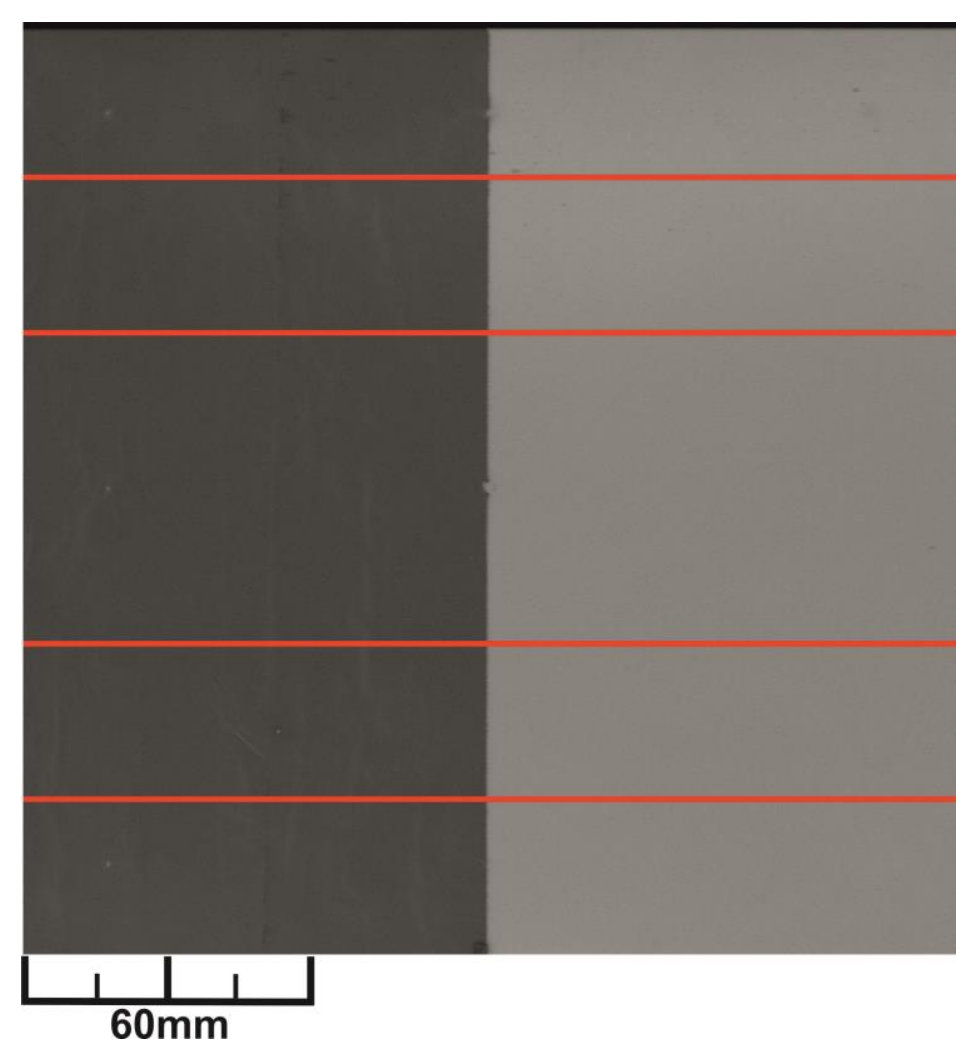

(a) Five scan lines for spatial resolution analysis

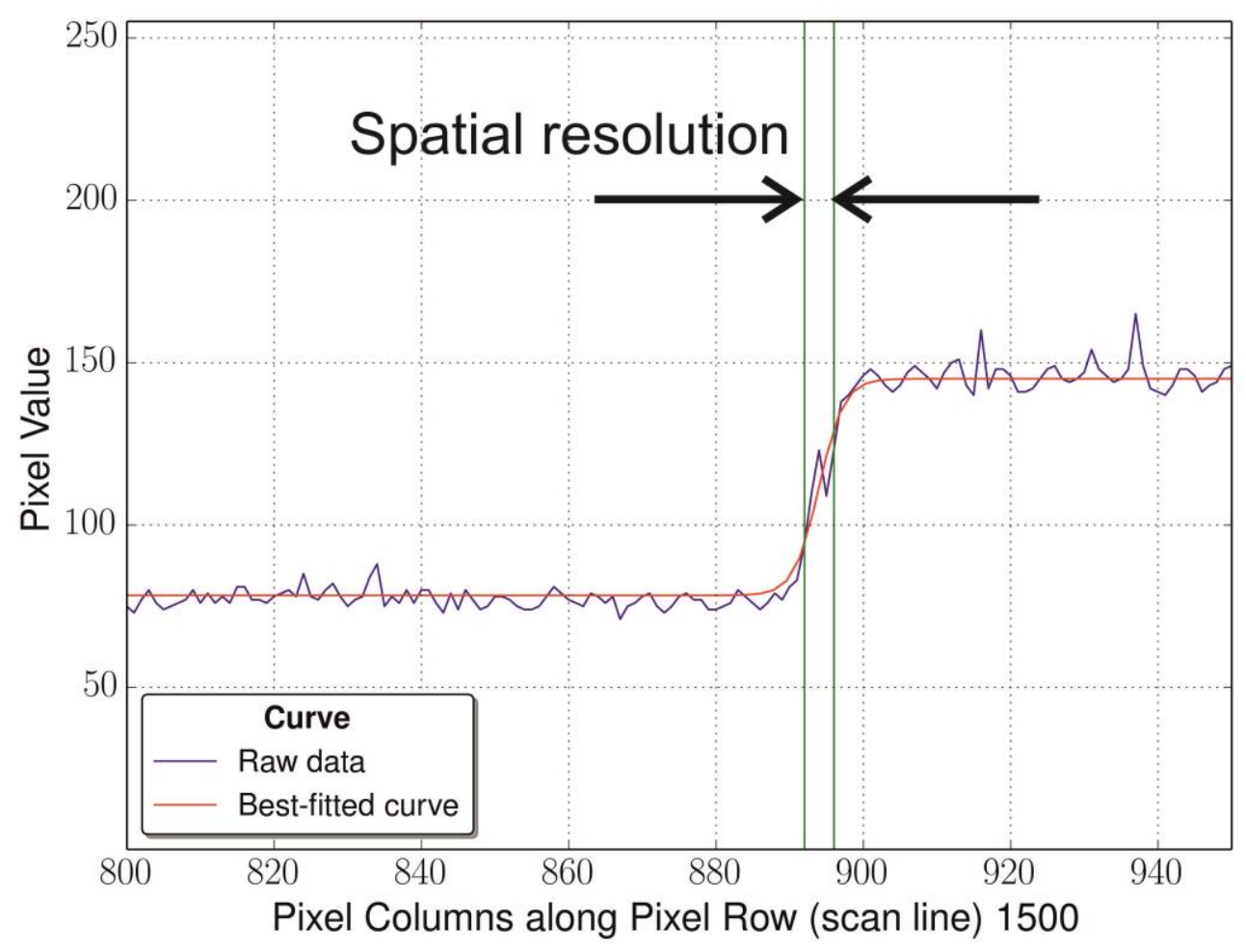

(b) Scan line pixel value ESF and the spatial resolution width

Fig. 7 Experimental result analysis 


$$
I(x)=A+B \tanh \left(\frac{x-C}{D}\right)
$$

Where

$\mathrm{I}(\mathrm{x})$ is the pixel value of the selected scan line (pixel row), $\mathrm{x}$ is the pixel column index of the selected scan line. "A"-"D" are parameters used to modify the standard $\tanh (\mathrm{x})$ function: " $\mathrm{A}$ " controls the offset in pixel value, "B" controls the amplification in magnitude, "C" controls the offset in pixel column index, and " $D$ " controls the spread of the function.

$$
R_{s p}=\Delta I m g_{C o l} \times \frac{I m g_{\text {width }, m m}}{I m g_{\text {width,pixel }}}
$$

Where

$\mathrm{R}_{\mathrm{sp}}(\mathrm{mm})$ is the pixel value, $\Delta \operatorname{Img}_{\mathrm{Col}}$ (pixel) is the number of pixels between two pixel columns with $25 \%$ and $75 \%$ of the maximum pixel values, $\operatorname{Img}_{\text {width,mm }}(\mathrm{mm})$ and $\operatorname{Img}_{\text {width,pixel }}$ are the electronic image 1D size (i.e. $60 \mathrm{~mm}, 180 \mathrm{~mm}$ and 1800 pixels).

Fig. 8 and Table 7 give the experimental and spatial resolution analysis results for electronic images with FOV of 180mm x 180mm, whilst Fig. 9 and Table 8 give results for images with FOV of $60 \mathrm{~mm} \times 60 \mathrm{~mm}$. Figs. 8(a) and 9(a) show that images with a FOV of $180 \mathrm{~mm} x$ $180 \mathrm{~mm}$ are less blurry and have greater image contrast. Moreover, Figs. 8(b) and 9(b) indicate that the pixel value profiles from images with a FOV of $180 \mathrm{~mm} \times 180 \mathrm{~mm}$ have greater Signal-Noise-Ratio (SNR). Fig. 10 visually presents the results summarised in Table 7 and 8. No observable trend can be drawn from Fig. 10, between data from the two different FOVs. 


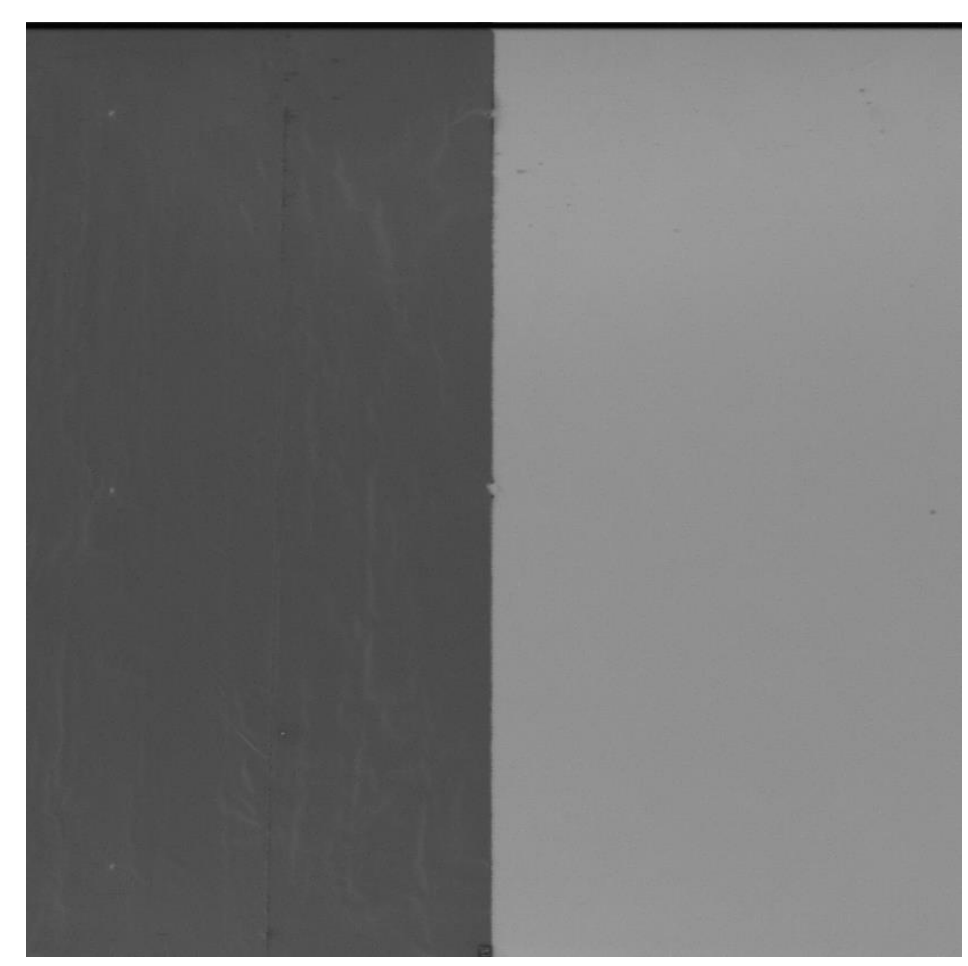

(a) Raw electronic image

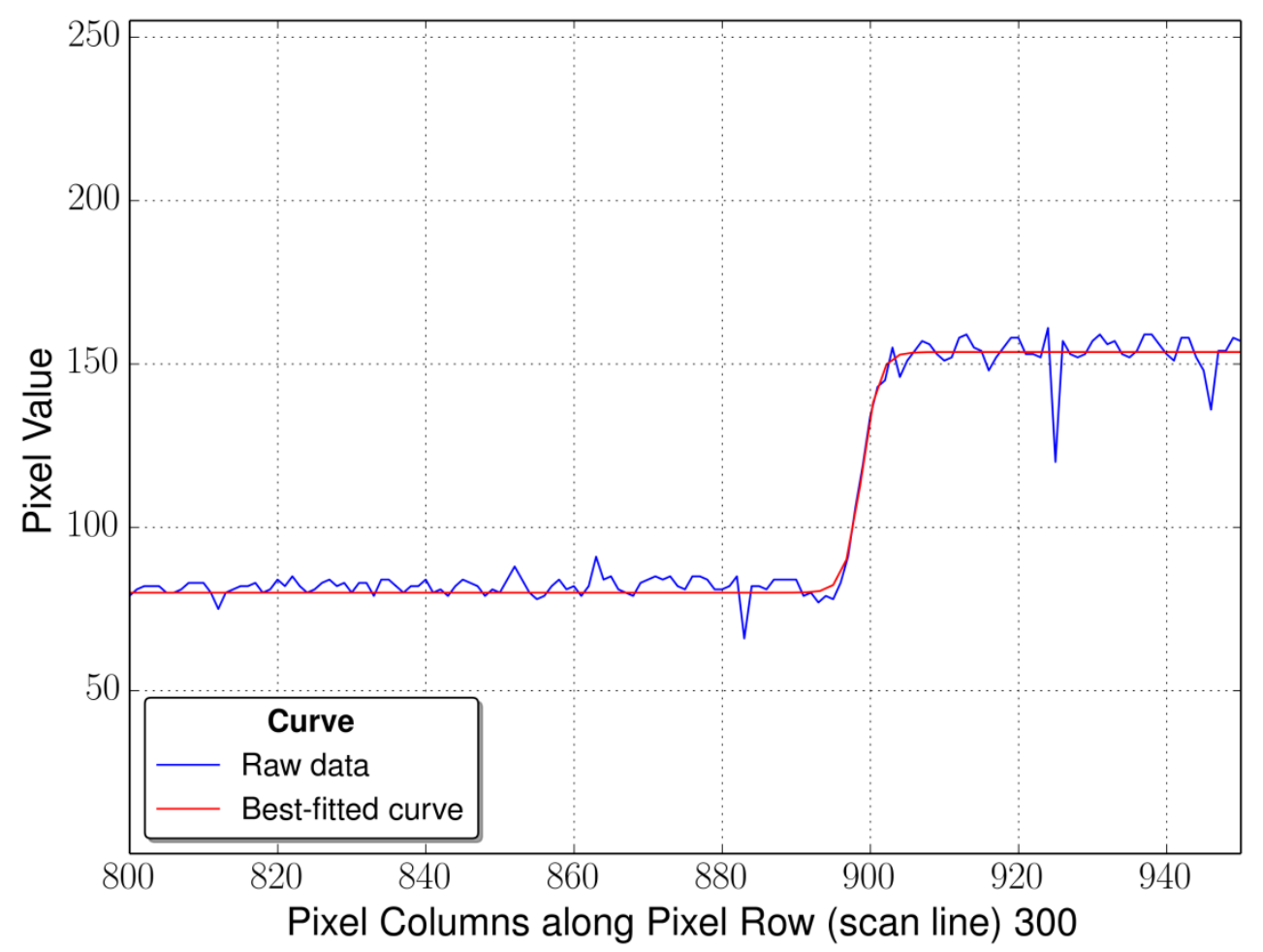

(b) Scan line pixel value ESF

Fig. 8 Typical results of images with FOV of $180 \mathrm{~mm} x 180 \mathrm{~mm}$ 
Table 7 Spatial resolution result (FOV: $180 \mathrm{~mm} \times 180 \mathrm{~mm}$ ). Data rounded to 3.s.f.

\begin{tabular}{ccc}
\hline $\begin{array}{c}\text { Electronic Image } \\
\text { Sample Number }\end{array}$ & $\mathrm{R}_{\mathrm{sp} / \mathrm{mm}}$ & Standard Error \\
\hline 1 & 0.350 & 0.0224 \\
2 & 0.275 & 0.0194 \\
3 & 0.300 & 0.0316 \\
4 & 0.325 & 0.0194 \\
5 & 0.350 & 0.0500 \\
\hline
\end{tabular}




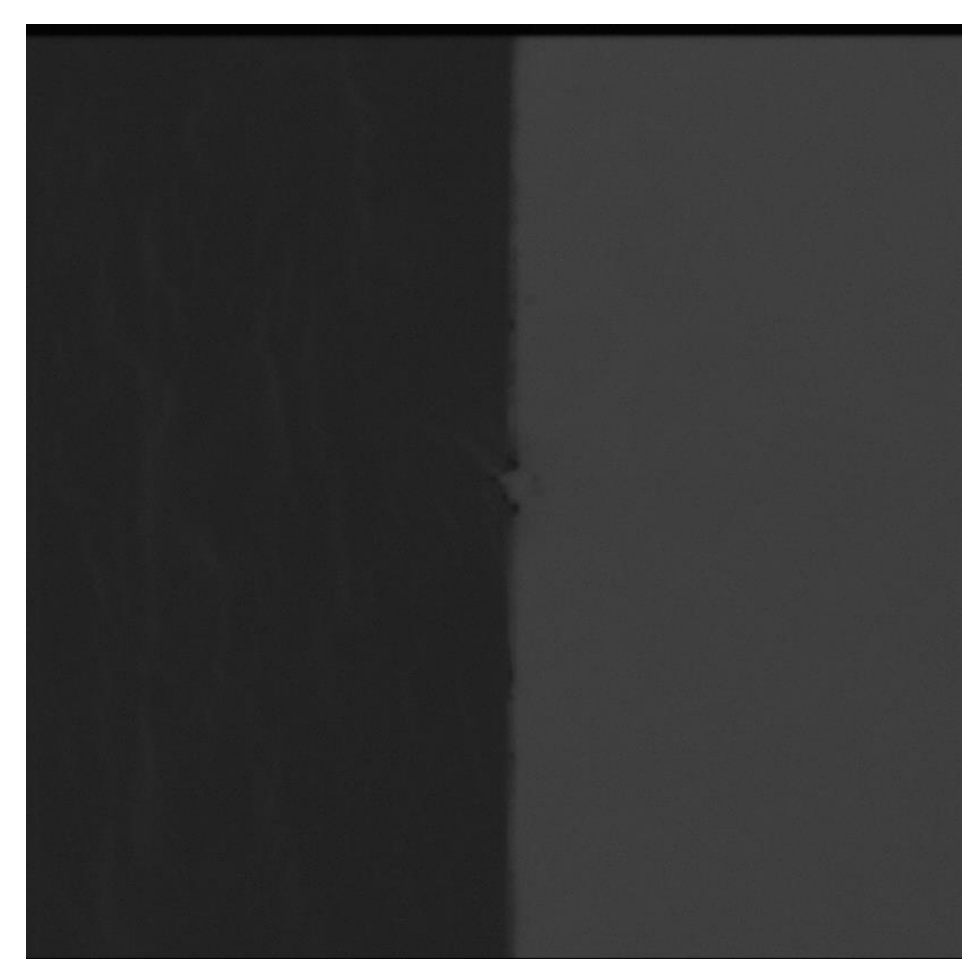

(a) Raw electronic image

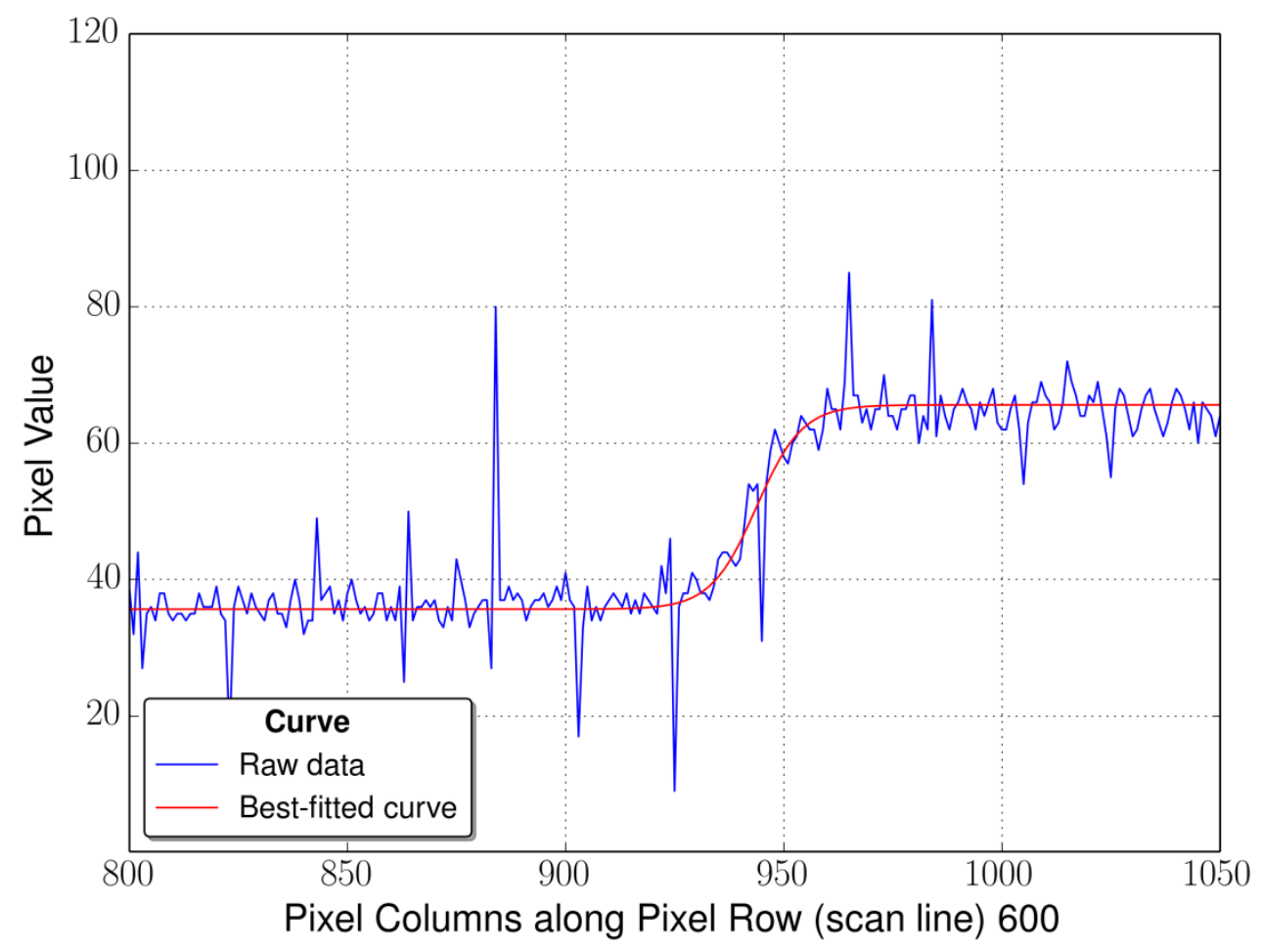

(b) Scan line pixel value ESF

Fig. 9 Typical results of images with FOV of $60 \mathrm{~mm} \times 60 \mathrm{~mm}$ 
Table 8 Spatial resolution result (FOV: $60 \mathrm{mmx} 60 \mathrm{~mm}$ ). Data rounded to 3.s.f.

\begin{tabular}{ccc}
\hline $\begin{array}{c}\text { Electronic Image } \\
\text { Sample Number }\end{array}$ & $\mathrm{R}_{\mathrm{sp} / \mathrm{mm}}$ & Standard Error \\
\hline 1 & 0.275 & 0.0286 \\
2 & 0.383 & 0.0373 \\
3 & 0.350 & 0.0373 \\
4 & 0.408 & 0.0194 \\
5 & 0.375 & 0.0286 \\
\hline
\end{tabular}

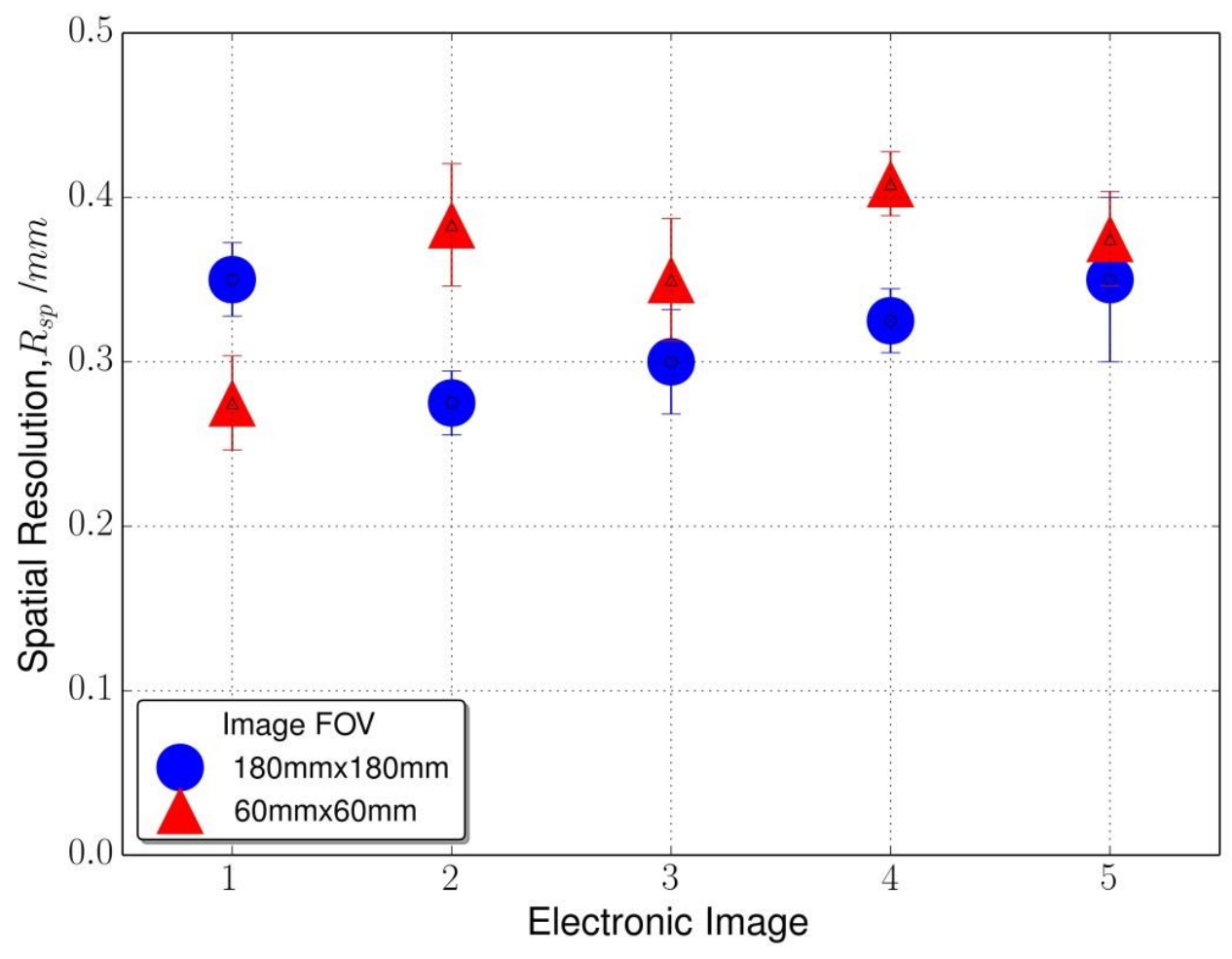

Fig. 10 Edge spatial resolution analysis result 


\section{Discussion}

\subsection{Differences in Electronic Image Quality with Different Image FOVs}

Differences in image contrast are observed from Figs. 8(a) and 9(a) whilst variations in SNR are seen from Figs. 8(b) and 9(b). It is postulated that the differences in image contrast and SNR both stem from the electron beam current settings when electronic imaging was carried out during the experiment. Table 5 shows that a beam current of $1 \mathrm{~mA}$ was used for images with FOV of $180 \mathrm{~mm} \times 180 \mathrm{~mm}$, whilst $0.5 \mathrm{~mA}$ was used for images with FOV of $60 \mathrm{~mm} \mathrm{x}$ $60 \mathrm{~mm}$. Table 5 also indicates that the image frame time was $27.3 \mathrm{~s}$ for all images regardless of their FOVs. The fixed image frame time led to more sever electron beam heating of the imaging target during the imaging of a smaller area. Heating of the target might lead to damage of the aluminium tape, and thus the beam current was set to $0.5 \mathrm{~mA}$ for the $60 \mathrm{~mm} \mathrm{x}$ $60 \mathrm{~mm}$ imaging area. The first drawback of this approach is that, the reduced primary machine electron beam current led to a reduction in feedback electron signal strength, leading to less image contrast for the electronic images with a FOV of $60 \mathrm{~mm} \times 60 \mathrm{~mm}$, i.e. Fig. 9(a) , when compared to images with a FOV of $180 \mathrm{~mm} \times 180 \mathrm{~mm}$, i.e. Fig. 8(a). In addition, the second drawback is the deterioration in the image signal SNR. It is thought that the level of noise stayed constant during the generation of electronic images with FOVs of $180 \mathrm{~mm} \mathrm{x}$ $180 \mathrm{~mm}$ and $60 \mathrm{~mm} \times 60 \mathrm{~mm}$. With a reduction in the feedback electron signal strength due to a smaller primary electron beam current, the SNR reduced, as observed when comparing Fig. 8(b) with Fig. 9(b).

Fig. 9(a) suffered from image blurriness when compared to Fig. 8(a). It is thought that the root cause lies in the limitation in spatial resolution. In theory, if the machine primary electron beam is infinitely small, the image spatial resolution would increase with pixel resolution, i.e. an increase in magnification. However, in reality, as demonstrated in Fig. 9(a), the image spatial resolution cannot keep up with the increase in magnification (indicated in Table 4). When pixel resolution becomes finer than the machine electron beam size, electron images became visually blurry.

\subsection{Spatial Resolution - Estimation and Experimental Results}

Referring to Tables 7 and 8, the average spatial resolution estimated for images with an FOV of $180 \mathrm{~mm} \times 180 \mathrm{~mm}$ and $60 \times 60 \mathrm{~mm}$ are $0.320 \mathrm{~mm}$ ( 3 s.f.) and $0.358 \mathrm{~mm}$ ( 3 s.f.). Firstly, these 
values are of the same order of magnitude when compared with the minimum achievable beam size of $0.2 \mathrm{~mm}$ claimed by Arcam AB [23] and $0.382 \mathrm{~mm}$ estimated in Table 3. This verifies the validity of the experimental setup and data analysis method.

Secondly, results from Tables 7 and 8 show that when interfaced with the Arcam A1 EBM machine, even with the machine limitation on beam size, with the measured spatial resolution of the order of $0.3 \mathrm{~mm}-0.4 \mathrm{~mm}$, the prototype still has a potential to detect the upper range of typical in-layer porosity (between $0.05-0.5 \mathrm{~mm}[7]$ ).

Thirdly, it is thought that results imply that the impasse of the spatial resolving power of this electronic imaging prototype lies with the machine electron beam size. Table 4 shows that for images with FOV of $60 \mathrm{~mm} \times 60 \mathrm{~mm}$, the pixel resolution is $0.033 \mathrm{~mm}$. This indicates that if an infinitely small machine electron beam is available, the measured spatial resolution should be expected to be of the order of $0.033 \mathrm{~mm}$, rather than the observed $0.358 \mathrm{~mm}$ ( 3 s.f.). The issue with image blurriness discussed in Section 4.1 also supports this argument.

\section{Conclusions}

This article has proposed a new indicator for spatial resolution by taking into account the effect of BSE information depth on top of the size of an electron beam, for electronic imaging conducted inside an Arcam A1 EBM machine. Theoretical analysis of the achievable spatial resolution was carried out and $0.382 \mathrm{~mm}$ was estimated to be the resolution when considering the influence of the presence of helium gas at $2 \times 10^{-3}$ mbar inside the EBM machine chamber. In addition, this study also disseminated a method to carry out an edge resolution experiment with the use of a custom electronic imaging system prototype, operating at room temperature, inside an EBM machine. Spatial resolution was evaluated at two image FOVs, i.e. $180 \mathrm{~mm} x$ $180 \mathrm{~mm}$ and $60 \mathrm{~mm} \times 60 \mathrm{~mm}$, and the observed resolution were $0.320 \mathrm{~mm}$ and $0.358 \mathrm{~mm}$ respectively. This measured spatial resolving power of the order of $0.3 \mathrm{~mm}-0.4 \mathrm{~mm}$ is thought to have shown that electronic imaging has the potential to be used for in-process EBM monitoring to detect the typical $0.3 \mathrm{~mm}-0.5 \mathrm{~mm}$ in-layer porosity within EBM manufactured components. However, in order to realise this in-process monitoring potential of electronic imaging, further spatial resolution studies need to be carried out at elevated, in-process EBM temperatures for various popular AM materials. It is believed that this study has contributed to the on-going development of an effective in-process EBM monitoring system by providing 
a method to analyse and benchmark the achievable spatial resolution of an electronic imaging system, which operates inside an EBM machine at room temperature.

\section{Acknowledgements}

The Author(s) declare(s) that there is no conflict of interest. This research received no specific grant from any funding agency in the public, commercial, or not-for-profit sectors. The EBAM machine was purchased, in part from a grant received for the EPSRC Centre for Innovative Manufacturing in Additive Manufacturing.

\section{References}

1. I. Gibson, D.W. Rosen, B Stucker (2010) Additive Manufacturing Technologies. Springer, New York, pp. 126-128

2. G. Baudana, S. Biamino, D. Ugues, M. Lombardi, P. Fino, M. Pavese, C. Badini, (2016) Titanium Aluminides for Aerospace and Automotive Applications Processed by Electron Beam Melting: Contribution of Politecnico di Torino, Metal Powder Report, Volume 71, Issue 3, 2016, Pages 193-199, ISSN 0026-0657, http://dx.doi.org/10.1016/j.mprp.2016.02.058.

3. O.L.A Harrysson, O. Cansizoglu, D.J. Marcellin-Little, D.R Cormier, H.A West (2008) Direct Metal Fabrication of Titanium Implants with Tailored Materials and Mechanical Properties using Electron Beam Melting Technology, Materials Science and Engineering: C, Volume 28, Issue 3, 2008, Pages 366-373, ISSN 0928-4931, http://dx.doi.org/10.1016/j.msec.2007.04.022.

4. X Gong, T Anderson, K Chou (2014) Review on Powder-Based Electron Beam Additive Manufacturing Technology. Manufacturing Review 1 2. DOI: 10.1051/mfreview/2014001 
5. National Institutio of Standards and Technology, U.S. Department of Commerce (2013) Measurement Science Roadmap for Metal-Based Additive Manufacturing Workshop Summary Report, Pages 61-70,

https://www.nist.gov/sites/default/files/documents/el/isd/NISTAdd_Mfg_Report_FIN $\underline{\text { AL-2.pdf }}$

6. X. Tan, Y. Kok, S.B Tor, C.K Chua (2014) Application of Electron Beam Melting (EBM) in Additive Manufacturing of an Impeller, Proc. of the Intl. Conf. on Progress in Additive Manufacturing, doi:10.3850/978-981-09-0446-3 076

7. S. Everton, M. Hirsch, P. Stravroulakis, R. Leach, A. Clare (2016) Review of In-Situ Process Monitoring and In-Situ Metrology for Metal Additive Manufacturing, Materials and Design 95 (2016) pp. 431-445, http://dx.doi.org/10.1016/i.matdes.2016.01.099

8. W.J. Sames V (0215) Additive Manufacturing of Inconel 718 Using Electron Beam Melting: Processing, Post-Processingm and Mechanical Properties, PhD Thesis, Texas A\&M University

9. M. Mani, B. Lane, A. Donmez, S. Feng, S. Moylan, R. Fesperman (2015) NISTIR 8036. Measurement Science Needs for Real-time Control of Additive Manufacturing Powder Bed Fusion Processes. http://dx.doi.org/10.6028/NIST.IR.8036

10. C. Arnold, C. Pobel, F. Osmanlic, C. Körner (2018) Layerwise monitoring of electron beam melting via backscatter electron detection. Rapid Prototyping Journal 24/8 (2018) 1401-1406. DOI 10.1108/RPJ-02-2018-0034

11. H. Wong, D. Neary, S. Shahzad, E. Jones, P. Fox, C. Sutcliffe (2018) Pilot Investigation of Feedback Electronic Image Generation in Electron Beam Melting and its Potential for In-Process Monitoring, Elsevier Journal of Materials Processing Technology, DOI: 10.1016/j.jmatprotec.2018.10.016

12. H. Wong, D. Neary, E. Jones, P. Fox, C. Sutcliffe (2018) Pilot capability evaluation of a feedback electronic imaging system prototype for in-process monitoring in electron beam additive manufacturing, Springer International Journal of Advanced Manufacturing Technology, DOI: 10.1007/s00170-018-2702-6 
13. H. Wong, D. Neary, S. Shahzad, E. Jones, P. Fox, C. Sutcliffe (2018) Pilot Feedback Electronic Imaging at Elevated Temperatures and its Potential for In-Process Electron Beam Melting Monitoring, Elsevier Additive Manufacturing (in-press)

14. G.F. Lorusso, D.C Joy (2003) Experimental Resolution Measurement in Critical Dimension Scanning Electron Microscope Metrology, SCANNING VOL. 25, pp. $175-180(2003)$

15. L. Reimer (1998) Scanning Electron Microscopy: Physics of Image Formation and Microanalysis, Springer, pp. $4-5$

16. A. Kaur, C. Ribton, W. Balachandaran (2015) Electron Beam Characterisation Methods and Devices for Welding Equipment, Elsevier Journal of Materials Processing Technology, http://dx.doi.org/10.1016/j.jmatprotec.2015.02.024

17. J.W. Elmer (2009) Characterization of Defocused Electron Beams and Welds in Stainless Steel and Refractory Metals using the Enhanced Modified Faraday Cup Diagnostic, LLNL-TR-410110, DOI: 10.2172/947235

18. A. Stratan, A. Zorila, L. Rusen,G. Nemes (2014) Measuring Effective Area of Spots From Pulsed Laser Beams, Optical Engineering 53(12), 122513, DOI: $10.1117 / 1 . O E .53 .12 .122513$

19. M.A. C. de Araújo, R. Silva, E. de Lima, D.P. Pereira, P.C. de Oliveira1 (2009) Measurement of Gaussian Laser Beam Radius using the Knife-Edge Technique: Improvement on Data Analysis, APPLIED OPTICS Vol. 48, No. 2

20. Course PH5060, Teaching Materials, Department of Physics, Indian Institute Of Technology Madras, Chennai, http://www.physics.iitm.ac.in/ ph5060/manuals/Gaussianlaserbeam.pdf

21. J. Tragardh, K. Macrae, C. Travis, R. Amor, G. Norris, S.H. Wilson, G.L. Oppo, G. Mcconnell (2015) A Simple but Precise Method for Quantitative Measurement of the Quality of the Laser Focus in a Scanning Optical Microscope, Journal ofMicroscopy, Vol. 259, Issue 12015 , pp. 66-73, doi: 10.1111/jmi.12249 
22. G.D Archard (1961) Back Scattering of Electrons, Journal of Applied Physics 32, 1505 (1961); doi: 10.1063/1.1728385

23. Arcan A1 EBM Machine Specification, http://www.arcam.com/wp-content/uploads/Arcam-A1.pdf

24. P. Wang, W. Sin, M.L. Nai, J. Wei (2017) Effects of Processing Parameters on Surface Roughness of Additive Manufactured Ti-6Al-4V via Electron Beam Melting, Materials 2017, 10, 1121; doi:10.3390/ma10101121

25. G.D. Danilatos (1988) Foundations of Environemtal Scanning Electron Microscopy, Advances in Electronics and Electron Physics, Vol. 71, Academic Press, Inc. ISBN 012-014671-1, pp. 162

26. A. Zoukel,L. Khouchaf, J.Di. Martino, D. Ruch (2012) Skirting effects in the variable pressure scanning electron microscope: Limitations and improvements, Micron 44 (2013) pp. 107-114, http://dx.doi.org/10.1016/j.micron.2012.05.004

27. L. Reimer (1998) Scanning Electron Microscopy: Physics of Image Formation and Microanalysis, Springer, pp. 135-148

28. D. Auroux (2010) 18.02SC Multivariable Calculus. Massachusetts Institute of Technology: MIT OpenCourseWare, https://ocw.mit.edu. License: Creative Commons BY-NC-SA. Course notes on "Least Square Interpolation” 